\title{
Effects of various factors on biomass, bioethanol, and biohydrogen production in green alga Chlamydomonas reinhardtii
}

\author{
Narravula Raga Sudha ${ }^{1}$, Duddela Varaprasad ${ }^{1}$, Pallaval Veera Bramhachari², Poda Sudhakar ${ }^{3}$, Thummala Chandrasekhar ${ }^{\text {* }}$ \\ ${ }^{1}$ Department of Environmental Science, Yogi Vemana University, Kadapa, A.P, India. \\ ${ }^{2}$ Department of Biotechnology, Krishna University, Machilipatnam, A.P, India. \\ ${ }^{3}$ Department of Biotechnology, Acharya Nagarjuna Univerisity, Guntur, A.P, India.
}

\begin{tabular}{l}
\hline ARTICLE INFO \\
\hline Article history: \\
Received on: March 13, 2021 \\
Accepted on: May 11, 2021 \\
Available online: September 01, 2021 \\
\hline
\end{tabular}

Key words:

Algae, cobalt, biomass, Tris base, ethanol, hydrogen

\begin{abstract}
Extensive usage of nonrenewable fossil fuels has urged the community to look into alternate renewable energy such as biofuels. The production of biofuels, including bioethanol, biohydrogen, biodiesel, etc., from crop plants again leads to food insecurity. Hence, the usage of algae, particularly green algae, is the best alternatives to generate biofuels. In the present study, the effects of various factors involved in algal growth in turn bioethanol and biohydrogen production levels were established by choosing green alga Chlamydomonas reinhardtii. Specifically, we crosschecked the effect of heavy metal Cobalt (Co), sodium bicarbonate, and Tris-acetate-phosphate (TAP) medium without Tris base on growth and biofuel production from C. reinhardtii under in vitro conditions. In the current work, $0.2 \mathrm{mg} / \mathrm{l} \mathrm{Co}$ in TAP medium enhanced the growth and biomass in C. reinhardtii cultures. Furthermore, ethanol production levels were improved in feedstocks grown in TAP medium with $2.0 \mathrm{mg} / 1$ sodium bicarbonate. In another experiment, hydrogen production was high in full TAP medium with Tris (trisaminomethane) base when compared to TAP without Tris base. In conclusion, this work may be useful to improve the biomass and biofuel production in green algae.
\end{abstract}

\section{INTRODUCTION}

The global demand for renewable energy is increasing day by day due to the continuous reduction in fossil fuels. Population explosion and their energy necessities is the main reason for overexploitation of nonrenewable fuel resources [1]. Specifically, for the past two centuries, people are completely dependent on fossil fuels without looking out for future shortages [2]. Hence, the production of renewable energy has become inevitable for sustainable development in the energy sector. On the contrary, the generation of renewable energy is one of the challenging issues in the entire world and it also decides the economy of a country [3]. Solar energy, hydro energy, wind energy, bioenergy, etc., are the main components of renewable energy and, at present, people are dependent on these kinds of resources [4]. However, the usage of renewable energy, including bioenergy, is increasing continuously in certain countries and the percentage of electricity generation from biofuels is also improving regularly [5].

\footnotetext{
*Corresponding Author

Thummala Chandrasekhar, Department of Environmental Science, Yogi Vemana University, Kadapa,A.P, India.E-mail: tcsbiotech@gmail.com
}

The production of biofuels and their content completely depends on the growth and biomass of the species. Biomass is nothing but organic matter in the form of leaves, stems, seeds, and all the other forms of advanced plants and whole cells of primitive plants such as algae [6,7]. Apart from these basic needs, biomass offers other significant environmental and consumer benefits, such as improving forest health, protecting air quality, and also offering the most dependable renewable energy. There are four generations of biofuels, i.e., in the first generation, the fuel was produced from food crops, including corn and maize, which again lead to food insecurity [8]. Later, second generation fuel was produced from nonfood crops and waste. But in the third generation, fuel was produced by improved biomass through advanced technology or algae/bacteria. In the fourth generation, fuels were also made from recent technology using nonarable land which includes electrofuels and photobiological solar fuels [9]. Furthermore, in advanced biofuel technologies, algae/microbes are grown in special bioreactors which are supplied with nutrients, waste organic material, and $\mathrm{CO}_{2}$ from the air or from conventional gas plants $[10,11]$. As they grow well in the reactors, it is easy to collect fuel from the bioreactor, but in some cases the downstream processing 
is expensive. At initial stages, the advanced plants were used for the production of biofuels [12]. Later, green algae were used as food, fodder, medicine, and nutraceuticals. However, in certain places, green algae were used as biofuel production [13-15].

The improvement of biomass in turn bioproducts in different edible and energy crops including algae is the present scenario in entire world. Algae belong to primitive plants that exist in both aquatic and terrestrial environments [16]. In certain old classification, these belong to a group of microorganisms due to their unicellular nature. But the algae group possesses both unicellular and multicellular organisms and adapt to autotrophic, heterotrophic, or mixotrophic lifestyles [1]. In another classification, algae are divided into green algae (chlorophyta), blue-green algae (cyanobacteria), red algae (rhodophyta), brown algae (phaeophyta), etc. Green algae are said to be ancestral to land plants having efficient photosynthetic capacity apart from photosynthetic bacteria [17]. Several advanced biotechnological methods were applied to enhance the biomass in plants and algae by altering the macronutrients and micronutrients, growth hormones, vitamins, etc. [18-20]. On the contrary, many green algae belonging to either fresh water or marine habitats remain as waste and are unused in certain countries.

Hence, in the present work, an attempt has been made to check the growth (biomass) and biofuel production in fresh water green alga Chlamydomonas reinhardtii using Cobalt (Co), sodium bicarbonate, and tris-acetate-phosphate (TAP) medium without the Tris base under in vitro conditions. Co is generally considered as toxic heavy metal which is available as complex form. It is indirectly involved in nitrogen metabolism but shows cytotoxic nature in certain advanced plants [21]. Sodium bicarbonate $\left(\mathrm{NaHCO}_{3}\right)$ is an inorganic carbon source which was used along with the TAP medium for improvement of biomass in certain species [22]. In addition, the importance of Tris base in the TAP medium is also emphasized for growth and photobiological hydrogen production which is useful in maintaining the $\mathrm{pH}$ in the medium [23]. So, with the aim to know biomass accumulation in turn biofuel production in $C$. reinhardtii, we treated the algal culture with the above mentioned metal/compounds under in vitro conditions.

\section{MATERIALS AND METHODS}

Green alga, C. reinhardtii, was collected and preserved as per the standard procedure in glycerol solution for future usage. For in vitro cultures, the TAP medium was prepared with or without Tris base to check the importance of growth in turn biological hydrogen production. In addition, TAP along with various concentrations of sodium bicarbonate $(0.0,2.0,4.0$, and $8.0 \mathrm{mg} / \mathrm{l})$ media were prepared and designated as CR0, CR1, CR2, and CR3 to know the feedstock capacity in ethanol production. Similarly, TAP along with various concentrations of Co chloride $(0.0,0.2,0.4,0.8,1.6$, and $3.2 \mathrm{mg} / \mathrm{l})$ media were also prepared. All the media were maintained with pH 7.0 using a pH meter (Elico limited, LI 617, Hyderabad, India) and kept in an autoclave for 20 minutes in $15 \mathrm{lbs}$ at $121^{\circ} \mathrm{C}$ (Inlab Equipment, Chennai, India). After completion of autoclave, media were allowed to cool down and then algal inoculation was carried out in a laminar air flow chamber (Hitech products, Chennai, India). Later, all the inoculated samples were kept in an orbital shaker (Remi Elektrotechnik Limited, Vasai, India) at $120 \mathrm{rpm}$ with continuous light conditions for further growth to reach log phase (around 4 days). Furthermore, algal samples were taken out at the $\log$ or early stationary phase and estimated the biomass using cell count which is crucial for the present study. Figure 1 shows the preparation of bioethanol and biohydrogen from algal samples.

Initial chlorophyll estimation was carried out with log phase cells using the modified Arnon's method [24]. For ethanol production, the early stationary phase of $C$. reinhardtii cultures was removed and boiled at $100^{\circ} \mathrm{C}$ for 90 minutes on a hot plate and later at room temperature $\alpha$-amylase was added and kept as standby for 90 minutes for carbohydrate extraction as per Sulfahri et al. [25]. Furthermore, the liquid was centrifuged at 5,000 rpm for 15 minutes and the supernatant was taken out and used in the fermentation process. The yeast peptone medium was prepared to maintain

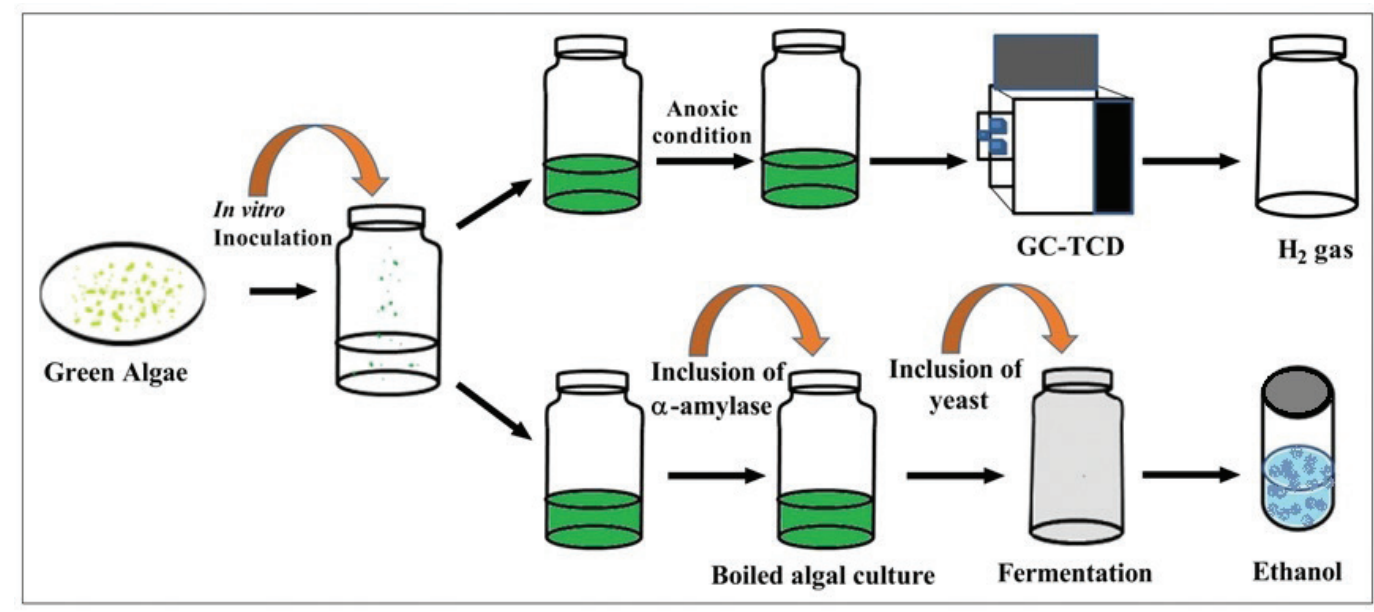

Figure 1. Schematic representation of hydrogen (upper) and ethanol (lower) production from C. reinhardtii. (Hydrogen) After reaching the log phase, equal biomass algal cells were purged with nitrogen to remove oxygen and allowed to grow in anoxic conditions. Later, hydrogen estimated using GC-TCD by using gaseous phase. (Ethanol) Before reaching the stationary phase, all the algal cells were boiled and added to the $\alpha$-amylase enzyme to breakdown the sugars. Finally, these sugars were fermented using yeast cells to generate ethanol. 
commercially available Baker's yeast (Saccharomyces cerevisiae) which is useful for the fermentation process. Later, algal feedstock solution along with $10 \%$ yeast mixture in a vial was closed tightly with a sealer. All the cultures were made oxygen-free environments with nitrogen gas. Furthermore, the cultures were kept in an orbital shaker at $120 \mathrm{rpm}$ with $27^{\circ} \mathrm{C}$. The determination of ethanol level was carried out using modified potassium dichromate method [26] and optical density readings were taken for $0,12,24,48,72$, and 96 hours at $660 \mathrm{~nm}$ with UV spectrophotometer (Shimadzu UV-800, India). The standard curve was made with commercially available $100 \%$ ethanol at different concentrations.

For biological hydrogen production, algal samples were taken out at the log phase and later equal biomass cultures were subcultured and finally purged with nitrogen. Quantification of $\mathrm{H}_{2}$ was carried out using gas chromatography-thermal conductivity detector (GCTCD; Shimadzu, GC-2014, Japan) at different time intervals [27]. The total volume of $\mathrm{H}_{2}$ was calculated based on the peak area and the statistical analysis was carried out using the standard Excel program.

\section{RESULTS AND DISCUSSION}

In the present work, experiments were initiated under in vitro conditions to standardize the optimum conditions for improvement of biomass in C. reinhardtii using the TAP medium with or without Co to check the growth and biomass levels. Furthermore, we screened sodium bicarbonate along with the TAP medium and also TAP with or without Tris base to know the ethanol and hydrogen production levels. All experiments were conducted at culture room conditions to know the effects of these nutrients, and the results obtained are documented below.

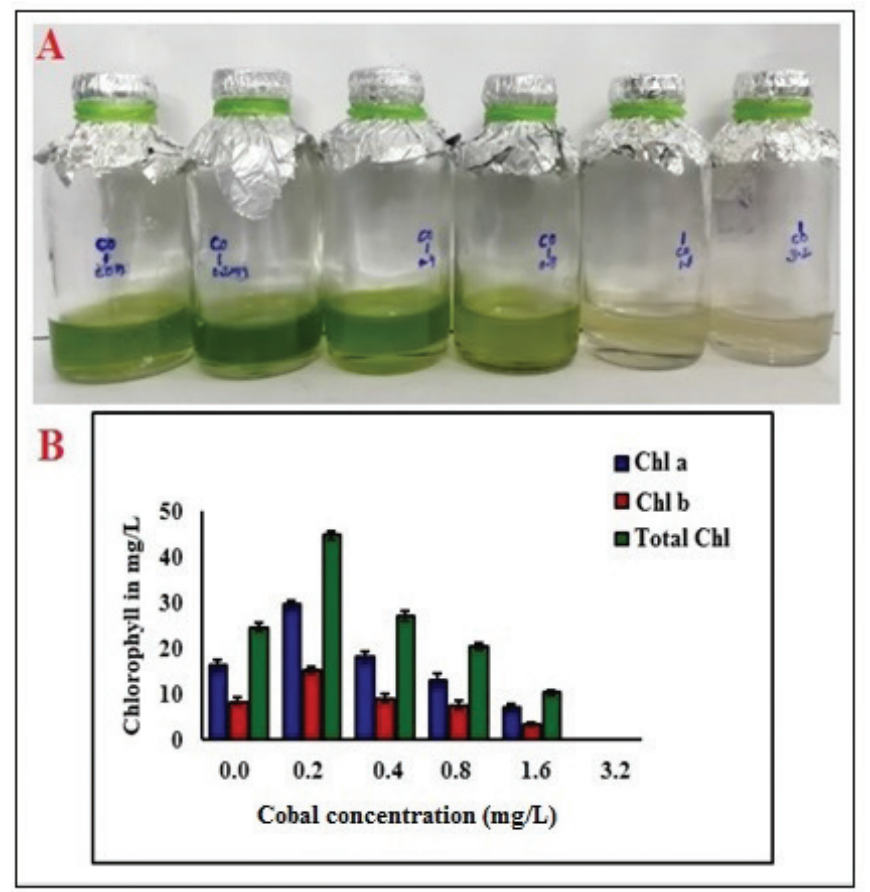

Figure 2. Growth and chlorophyll estimation in $C$. reinhardtii cultures treated with various concentrations of Co. (A) Growth of the culture and (B) chlorophyll estimation. In figure $\mathrm{B}, x$-axis indicates the Co concentrations and $y$-axis indicates the chlorophyll (biomass) concentrations.

\subsection{Impact of Co on Growth and Biomass Accumulation in $C$. reinhardtii}

Co is one of the important heavy metals to study for advanced plants as well as for algae to check their growth and development. Interestingly, TAP with $0.2 \mathrm{mg} / 1$ of $\mathrm{Co}$ augmented the growth and biomass (total chlorophylls) of $C$. reinhardtii cultures when compared to untreated cultures (Fig. 2A and B).

On the contrary, higher concentrations of Co inhibit the growth and biomass accumulation. Specifically, absolute inhibition of growth was noted at $3.2 \mathrm{mg} / \mathrm{l}$ Co treatment. Cavusoglu and Yalcin [21] also noted the cytotoxic nature of Co through lipid peroxidation of plant cells. Similar toxic trends were also noted in the accumulation of chlorophyll contents (biomass) in this algal culture (Fig. 2B). Chlorophyll content declined progressively with increasing concentrations of the heavy metal.

\subsection{Optimal Sodium Bicarbonate Enhances the Growth in turn Ethanol Content in $C$. reinhardtii}

Different concentrations of sodium bicarbonate, i.e., 2.0, 4.0, and $8.0 \mathrm{mg} / 1$ were added to the TAP medium and algal cultures were maintained under in vitro conditions. Optimal growth was observed after 3 days of incubation in TAP with $2.0 \mathrm{mg} / 1$ sodium bicarbonate $(\mathrm{CR} 1)$ in $C$. reinhardtii when compared to $\mathrm{CR} 0$ (without sodium bicarbonate) medium.

In agreement with visual observation, the amount of ethanol production in the fermentation process was also high in the feedstock grown in CR1 medium at 72 hours (Fig. 3). Hence, we conclude that sodium bicarbonate acts as carbon source in the algal culture up to some extent and the critical level is $2.0 \mathrm{mg} / \mathrm{l}$. Probably, feedstock grown in CR1 medium possess a high content or suitable sugars for ethanol generation. On the contrary, higher amounts of sodium bicarbonate (CR2 and CR3) inhibited the growth in turn ethanol content. Similarly, Mankee and Keatteong

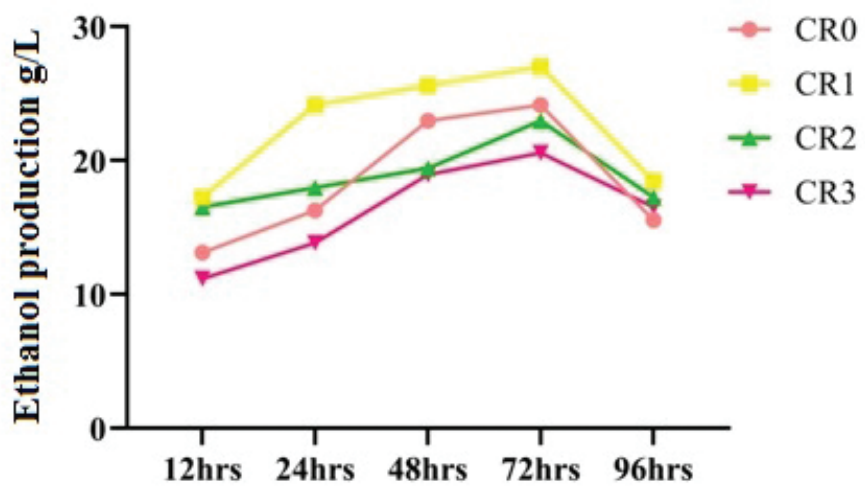

Time in hrs

Figure 3. Ethanol production using various feedstocks of $C$. reinhardtii cultures grown in TAP with various concentrations of sodium bicarbonate. (CR0) without sodium bicarbonate, (CR1) with $2.0 \mathrm{mg} / 1$ sodium bicarbonate, (CR2) with $4.0 \mathrm{mg} / 1$ sodium bicarbonate, and (CR3) with $8.0 \mathrm{mg} / 1$ sodium bicarbonate). In figure, $x$-axis indicates the fermentation time intervals and $y$-axis indicates the ethanol production levels. The data includes three replicates $(n=3)$. 


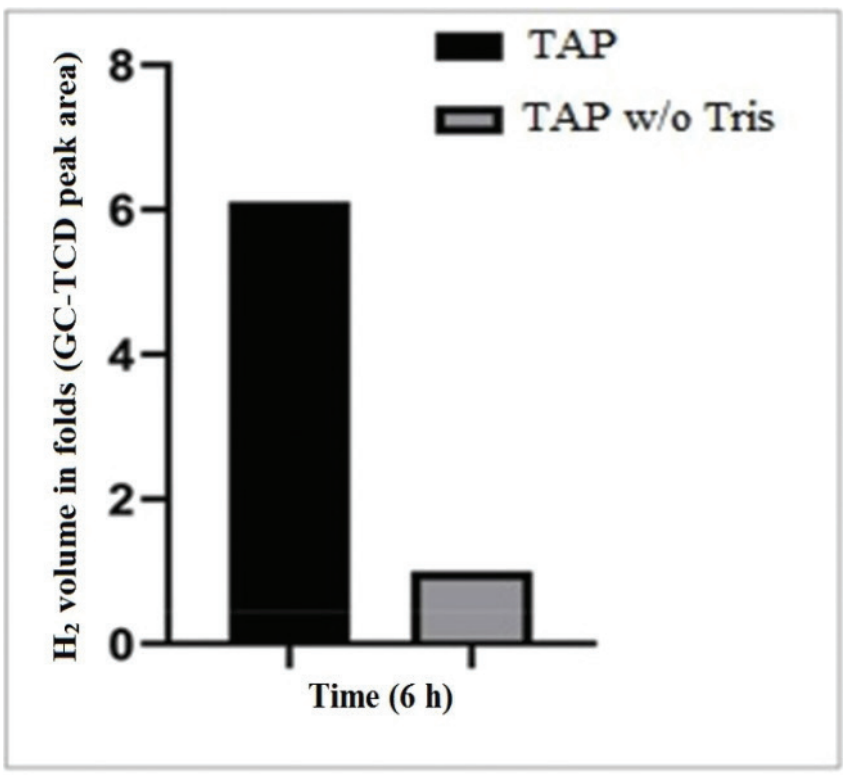

Figure 4. Photobiological hydrogen production using full TAP and TAP without Tris base (TAP w/o Tris) media. In figure, $x$-axis indicates the data collection time and $y$-axis indicates the hydrogen volume in folds.

[28] and Mokashi et al. [22] also emphasized the role of sodium bicarbonate on algal growth and biomass contents.

\subsection{Full TAP Enhances the Growth in turn Hydrogen Content in $C$. reinhardtii}

After 3 days of incubation in an orbital shaker, the log phase cultures were used for estimation of biological hydrogen production. Cultures purged with nitrogen (to remove oxygen) were kept again for estimation of the hydrogen levels. Cultures grown in a full TAP medium exhibited better hydrogen levels when compared to TAP without Tris base (TAP w/o Tris) after 6 hours (Fig. 4).

These data indicate the importance of Tris base for maintenance of $\mathrm{pH}$ in algal cultures. Similarly, Paramesh et al. [11], Juneja et al. [19], Granum and Myklestad [23], and Ghalanbor et al. [29] worked on $\mathrm{pH}$ maintenance in algal cultures to enhance yield levels.

\section{CONCLUSION}

Recently, the algal group is one of the best alternatives to produce food products, medicine, and biofuels. In the present investigation, algal biomass and both ethanol and hydrogen production levels were estimated using heavy metal Co, sodium bicarbonate, and strength of the TAP medium. TAP with 0.2 $\mathrm{mg} / \mathrm{l}$ of Co improved the growth and biomass in $C$. reinhardtii cultures. In addition, ethanol production levels were enhanced in feedstocks grown in TAP with $2.0 \mathrm{mg} / 1$ of sodium bicarbonate (CR1) medium. Full-strength TAP medium with Tris base augmented the biological hydrogen production. This work may be useful to improve the biomass in various algal species in turn biofuel generation.

\section{ACKNOWLEDGMENTS}

The authors are thankful to Dr. K. Paramesh, Dr. LV Reddy, and Prof. MV Shankar, Yogi Vemana University, India, for providing yeast, GC-TCD work, and technical help.

\section{ETHICAL APPROVAL}

Not applicable for this work.

\section{CONFLICT OF INTEREST}

The authors declare that there is no conflict of interest in this work.

\section{FUNDING}

There is no funding to report for this work.

\section{REFERENCES}

1. Melis A, Zhang L, Forestier M, Ghirardi ML, Seibert, M. Sustained photobiological hydrogen gas production upon reversible inactivation of oxygen evolution in the green alga Chlamydomonas reinhardtii. Plant Physiol 2000;122:127-36.

2. Das D, Veziroglu TN. Advances in biological hydrogen production processes. Int J Hydrogen Energy 2000;33:6046-57.

3. Govindjee. Matthias Rögner (ed). Biohydrogen. Photosynth Res 2015;124:337-9.

4. Ghirardi ML, Zhang L, Lee JW, Flynn T, Seibert M, Greenbaum E, et al. Microalgae: a green source of renewable $\mathrm{H}_{2}$. Trends Biotechnol 2000;18:506-11.

5. Nagarajan D, Lee DJ, Kondo A, Chang JS. Recent insights into biohydrogen production by microalgae from biophotolysis to dark fermentation. Bioresour Technol 2017;227:373-87.

6. Chartier P, Beenackers AACM, Grassi G. (eds.). Biomass for energy, environment, agriculture, and industry. Elsevier Science, Oxford, UK, 1995.

7. Varaprasad D, Ragasudha N, Parveen SN, Chandrasekhar, T. Effect of various solvents on chlorophyll and carotenoid extraction in green algae: Chlamydomonas reinhardtii and Chlorella vulgaris. Ann Plants Soil Res 2019;21:341-5.

8. Saladini F, Patrizi N, Pulselli FM, Marchettini N, Bastianoni S. Guidelines for emergy evaluation of first, second and third generation biofuels. Renew Sustain Energy Rev 2016;66:221-7.

9. Pugazhendhi A, Shobana S, Nguyen DD, Bhanu JR, Sivagurunathan P, Chang SW, et al. Application of nanotechnology (nanoparticles) in dark fermentative hydrogen production. Int J Hydrogen Energy 2019;44:431-40.

10. Guo Z, Li Y, Guo H. Effect of light/dark regimens on hydrogen production by Tetraselmis subcordiformis coupled with an alkaline fuel cell system. Appl Biochem Biotechnol 2017;183:1295-303.

11. Paramesh K, Lakshmana Reddy N, Shankar MV, Chandrasekhar T. Enhancement of biological hydrogen production using green alga Chlorococcum minutum. Int J Hydrogen Energy 2018;43:3957-66.

12. Cowie AL, Gardner WD. Competition for the biomass resource: greenhouse impacts and implications for renewable energy incentive schemes. Biomass Bioenergy 2007;31:601-7.

13. Khan MI, Shin JH, Kim JD. The promising future of microalgae: Current status, challenges, and optimization of a sustainable and renewable industry for biofuels, feed, and other products. Microb Cell Fact 2018;17:1-21.

14. Paramesh K, Chandrasekhar T. Improvement of photobiological hydrogen production in Chlorococcum minutum using various oxygen scavengers. Int J Hydrogen Energy 2020;13:7641-6. 
15. Kanygin A, Milrad Y, Chandrasekhar T, Kiera R, Baker P, Marco P, et al. Rewiring photosynthesis: photosystem I-hydrogenase fusion makes $\mathrm{H}_{2}$ in vivo. Energy Environ Sci 2020;13:2903-14.

16. Matsuo T, Ishiura M. Chlamydomonas reinhardtii as a new model system for studying the molecular basis of the circadian clock. FEBS Lett 2011;585:1495-502.

17. Yoon HS, Hackett JD, Ciniglia C, Pinto G, Bhattacharya D. A Molecular timeline for the origin of photosynthetic eukaryotes. Mol Biol Evol 2004;21:809-18.

18. Muehlbach HP. Use of plant cell cultures in biotechnology. Biotechnol Annu Rev 1998;4:113-76.

19. Juneja A, Ceballos RM, Murthy GS. Effects of environmental factors and nutrient availability on the biochemical composition of algae for biofuels production: a review. Energies 2013;6:4607-38.

20. Anto S, Mukherjee SS, Muthappa R, Mathimani T, Deviram G, Kumar SS, et al. Algae as green energy reserve: technological outlook on biofuel production. Chemosphere 2020;242:125079.

21. Cavusoglu K, Yalcin E. detection of lipid peroxidation and cytotoxicity induced by aluminium (Al) and cobalt (Co) ions in barbunia root tip cells. J Environ Biol 2011;5:661-6.

22. Mokashi K, Shetty V, George SA, Sibi G. Sodium bicarbonate as inorganic carbon source for higher biomass and lipid production integrated carbon capture in Chlorella vulgaris. Achiev Life Sci 216;10:111-7.

23. Granum E, Myklestad SM. A photobioreactor with $\mathrm{pH}$ control: demonstration by growth of the marine diatom Skeletone macostatum. J Plankton Res 2002;24:557-63.
24. Arnon D. Copper enzymes in isolated chloroplasts. Polyphenoloxidase in Beta vulgaris. Plant Physiol 1949;24:1-15.

25. Sulfahri MS, Amin M, Sumitro SB, Saptasari M. Bioethanol production from algae Spirogyra hyalina using Zymomonas mobilis. Biofuels 2016;7:621-6.

26. Caputi AJ, Ueda M, Brown T. Spectrophotometric determination of ethanol of wine. Am J Enol Viticulture 1968;19:160-5.

27. Lakshmana Reddy N, Emin S, Valant M, Shankar MV. Nanostructured $\mathrm{Bi}_{2} \mathrm{O}_{3} @ \mathrm{TiO}_{2}$ photocatalyst for enhanced hydrogen production. Int J Hydrogen Energy 2017;42:6627-36.

28. Mankee L, Keatteong L. Effect of carbon source towards the growth of Chlorella vulgaris for $\mathrm{CO} 2$ bio-mitigation and biodiesel production. Int J Greenh Gas Con 2013;14:169.

29. Ghalanbor Z, Ghaemi N, Marashi SA, Amanlou M, Habibi-Rezaei M, Khajeh K, et al. Binding of tris to Bacillus licheniformis $\alpha$-amylase can affect its starch hydrolysis activity. Protein Pept Lett 2008;15:212-4.

\section{How to cite this article:}

Sudha NR, Varaprasad D, Bramhachari PV, Sudhakar P, Chandrasekhar T. Effects of various factors on biomass, bioethanol, and biohydrogen production in green alga Chlamydomonas reinhardtii. J Appl Biol Biotech 2021; 9(05):152-156. 